\title{
Velocidade de ganho de peso e práticas alimentares no primeiro ano de vida em lactentes de baixo nível socioeconômico
}

\author{
Weight gain rate and feeding practices \\ of low-socioeconomic status infants
}

Samira Carvalho GONÇALVES'

Maria Laura da Costa LOUZADA ${ }^{1}$

Paula Dal Bó CAMPAGNOLO²

Márcia Regina VITOLO'

R E S U M O

\section{Objetivo}

Avaliar a velocidade do ganho de peso e sua associação com as práticas alimentares no primeiro ano de vida em crianças de baixo nível socioeconômico.

\section{Métodos}

Utilizaram-se dados de peso e comprimento ao nascer obtidos dos registros hospitalares e as medidas antropométricas aferidas aos 6 e 12 meses para o cálculo do ganho de peso no primeiro e no segundo semestres de vida. Dados das práticas alimentares das crianças foram obtidos por meio de entrevistas com as mães aos 6 e 12 meses de idade das crianças.

\section{Resultados}

Avaliaram-se 328 crianças: 184 meninos e 144 meninas. As meninas apresentaram maior ganho de peso em relação ao padrão da Organização Mundial de Saúde nos primeiros 6 meses de vida (4.452kg versus $4.079 \mathrm{~kg}, p=0,000)$. Dos 6 aos 12 meses, o ganho de peso das crianças avaliadas foi significativamente maior em comparação ao padrão da Organização Mundial de Saúde para os dois sexos (1.929kg versus $1.688 \mathrm{~kg}, p=0,000$ para os meninos e $1.900 \mathrm{~kg}$ versus $1.618 \mathrm{~kg}, p=0,001$ para as meninas). As crianças do sexo masculino que receberam aleitamento materno exclusivo por período menor que quatro meses apresentaram maior ganho de peso entre 6 e 12 meses de idade em relação àquelas que receberam somente leite materno por período igual ou superior a quatro meses ( $M=2,077, D P=0,777$ versus $M=1,814$, $\mathrm{DP}=0,669 p=0,02)$.

1 Universidade Federal de Ciências da Saúde de Porto Alegre, Departamento de Nutrição. R. Sarmento Leite, 245, 90050-170,

Porto Alegre, RS, Brasil. Correspondência para/Correspondence to: MR VITOLO.E-mail: <vitolo@ufcspa.edu.br>.

2 Universidade do Vale do Rio dos Sinos, Escola Superior de Saúde. São Leopoldo, RS, Brasil. 
556 | SC GONÇALVES et al.

\section{Conclusão}

Este estudo evidenciou que as crianças avaliadas apresentaram ganho de peso excessivo no primeiro ano de vida, e que o aleitamento materno exclusivo apresentou papel protetor.

Termos de indexação: Aleitamento materno. Ganho de peso. Ingestão de alimentos. Lactente.

\section{A B S T R A C T}

\section{Objective}

This study assessed weight gain rate during the first year of life of low-socioeconomic status infants and verified its association with feeding practices.

\section{Methods}

Weight gain during the first 6 and 12 months of life was calculated using birth weight and length data obtained from hospital records and anthropometric measurements done when the infants were 6 and 12 months old. Dietary data were collected during interviews with the mothers when the children were 6 and 12 months old.

\section{Results}

A total of 328 children were assessed, 184 boys and 144 girls. The girls gained more weight from birth to 6 months, as compared with the World Health Organization data $(4,452 \mathrm{~kg}$ versus $4,079 \mathrm{~kg} p=0,000)$. From 6 to 12 months of age, the children's weight gain was significantly higher than that of the World Health Organization data for both sexes $(1,929 \mathrm{~kg}$ versus 1,688 $\mathrm{kg}, p=0,000$, for boys and 1,900 kg versus 1,618kg, $p=0,001$, for girls). The boys who were exclusively breastfed for less than 4 months gained more weight from 6 to 12 months of age than those who were exclusively breastfed for 4 months or more $(M=2,077, S D=0,777$ versus $M=1,814, S D=0,669, p=0.02$ ).

\section{Conclusion}

The children in this study showed excessive weight gain during the first year of life. Exclusive breastfeeding was a protective factor.

Indexing terms: Breast feeding. Weight gain. Eating. Infant.

\section{N T R O D U Ç Ã O}

A obesidade infantil configura-se como emergente problema de saúde pública em todo o mundo ${ }^{1}$. A prevalência global de excesso de peso e de obesidade entre pré-escolares aumentou de 4,2\%, em 1990, para 6,7\%, em 2010, e a mudança foi relativamente maior em países em desenvolvimento ${ }^{2}$. No Brasil, a Pesquisa Nacional de Demografia e Saúde mostrou estabilidade na prevalência de excesso de peso para altura em crianças de 1996 a 2006³, mas estudos de base populacional em diversas regiões do país têm alertado para excesso de peso em crianças menores de cinco anos ${ }^{4-7}$.

Estudos recentes indicam que o ganho de peso excessivo no primeiro ano de vida é importante determinante do desenvolvimento de sobrepeso e de obesidade em pré-escolares, escolares e adolescentes de diferentes níveis socioeconômicos ${ }^{8-10}$. Além disso, o ganho de peso excessivo na infância está associado a alterações na distribuição de gordura corporal ${ }^{11,12}$ e à diminuição das concentrações corporais de grelina e adiponectina, o que pode contribuir para o desenvolvimento de doenças crônicas na vida adulta ${ }^{11}$.

O estado nutricional na infância está fortemente relacionado a fatores nutricionais e ambientais ${ }^{13-15}$, e sua evolução é potencialmente modificável pelas práticas alimentares ${ }^{16}$. O estudo multicêntrico da Organização Mundial da Saúde $(\mathrm{OMS})^{17}$ desenvolveu curvas de crescimento, desenvolvimento e ganho de peso de crianças com possibilidades de alcançarem pleno potencial genético de crescimento, ou seja, que vivem em 
condições sanitárias favoráveis e com padrões alimentares constituídos por aleitamento materno exclusivo por pelo menos quatro meses, introdução à alimentação complementar aos seis meses de vida e continuidade do aleitamento até pelo menos doze meses ${ }^{18}$. Dessa forma, o objetivo do presente estudo foi avaliar a velocidade do ganho de peso e sua associação com as práticas alimentares no primeiro ano de vida em crianças de baixo nível socioeconômico.

\section{MÉ T O D O S}

O presente estudo foi realizado com dados de um estudo maior, que se caracterizou por um ensaio de campo randomizado, cujos métodos e resultados principais foram detalhados anteriormente ${ }^{19}$. As mães e as crianças do grupo intervenção receberam dez visitas domiciliares durante o primeiro ano de vida das crianças, nas quais tiveram aconselhamento dietético baseado nos "Dez passos da alimentação saudável para crianças brasileiras menores de dois anos", enquanto aquelas pertencentes ao grupo-controle foram visitadas durante o primeiro ano de vida somente para realização da coleta de dados. Para o presente estudo, as análises foram realizadas agrupando-se as crianças que receberam a intervenção e aquelas consideradas do grupo-controle.

Entre outubro de 2001 e junho de 2002, pares de mãe-bebê foram recrutados na maternidade do Sistema Único de Saúde do Hospital Centenário da cidade de São Leopoldo (RS). Mães de crianças que nasceram a termo ( $\geq 37$ semanas) e com peso ao nascer $\geq 2.500 \mathrm{~g}$ foram identificadas e convidadas a participar do estudo. Os critérios de exclusão foram: mães HIV positivas, recém-nascidos com má-formação congênita ou encaminhados à Unidade de Terapia Intensiva (UTI) e parto múltiplo. Do total de mães convidadas, $89,5 \%(n=500)$ aceitaram ingressar do estudo.

Estudantes de graduação em Nutrição realizaram visitas domiciliares aos 6 e 12 meses de idade das crianças para obtenção das variáveis de interesse do estudo. A equipe de coleta de dados participou de sessões de treinamento para obtenção dos dados do questionário e das medidas antropométricas. Mensalmente, 10\% dos questionários completos foram selecionados aleatoriamente para confirmação da autenticidade dos dados por meio de ligações telefônicas para as mães.

O cálculo do tamanho da amostra foi realizado a priori e baseou-se no objetivo principal do projeto maior: aumento da prevalência de aleitamento materno exclusivo com a intervenção. Considerando-se frequência de aleitamento materno exclusivo até os quatro meses de $21,6 \%$ no grupo-controle, estimou-se diferença de 65,0\% na frequência dessa prática entre os grupos após a intervenção. Outros parâmetros para esse cálculo foram: poder de $80,0 \%$ e nível de confiança de $95,0 \%$, o que determinou um tamanho amostral de 363 crianças. Considerando uma previsão de perdas de $25,0 \%$, foram recrutados 500 pares mãe-criança. Para o presente estudo, utilizaram-se dados de 328 crianças aos 6 meses e 308 aos 12 meses de idade.

\section{Coleta de dados}

Dados de identificação e informaç̧ões para localização das residências das crianças na comunidade foram coletados no recrutamento. A partir dos registros do hospital, obtiveram-se dados de sexo, peso e comprimento ao nascer.

Foram realizadas visitas domiciliares quando as crianças completavam seis meses de idade e, nessa ocasião, as mães responderam ao questionário sobre características maternas e familiares, tais como: escolaridade e situação conjugal maternas, idade no nascimento da criança e renda familiar mensal. Na mesma ocasião, as mães responderam a perguntas sobre as práticas alimentares da criança até os 6 meses de idade. As seguintes variáveis foram investigadas: duração do aleitamento materno, idade de introdução de novos alimentos e bebidas, consumo de açúcar de adição, ingestão de leite de vaca e adição de açúcar e farinhas na mamadeira. Aleitamento 
materno exclusivo foi definido como o uso de aleitamento materno como único alimento para criança, sem o consumo de chá, água, outros líquidos ou sólidos, sendo exceção medicamentos e suplementos vitamínicos e minerais ${ }^{20}$.

No período em que as crianças completavam 12 meses, novas visitas domiciliares foram realizadas e as mães responderam sobre o consumo de leite de vaca, adição de farinha e açúcar nas mamadeiras no período de 6 a 12 meses, além do consumo de refrigerante no mês anterior à entrevista.

Aos 6 e aos 12 meses de idade das crianças, medidas antropométricas foram obtidas de acordo com técnicas padronizadas. As crianças foram pesadas sem roupas e sem fraldas em balança pediátrica (Techline ${ }^{\circledR}$, São Paulo, Brasil) devidamente tarada, e o comprimento foi aferido em decúbito dorsal, por meio de estadiômetro de madeira para uso pediátrico (Serwital ${ }^{\circledR}$, Porto Alegre, Brasil). Calculou-se o ganho de peso nos primeiros 6 meses de vida subtraindo-se do peso da criança aos 6 meses o peso da criança ao nascer. Repetiu-se o mesmo procedimento com o peso da criança aos 12 meses: subtraiu-se desse número o peso da criança aos 6 meses para avaliação do ganho de peso no segundo semestre de vida.

\section{Análise estatística}

Os dados foram submetidos à dupla digitação no programa Statistical Package for Social Sciences (SPSS) versão 11.0 (SPPS, Chicago, USA) e validados no programa Epi Info versão 6.4 (CDC, Atlanta, USA). O programa estatístico SPSS versão 16.0 foi utilizado para as análises estatísticas. Como este estudo utilizou dados de ensaio randomizado, avaliou-se a presença do efeito da intervenção sobre o desfecho do presente estudo - ganho de peso no primeiro ano de vida - com o objetivo de verificar a possibilidade de se conduzirem as análises por meio de agrupamento das crianças que receberam a intervenção e daquelas consideradas do grupo-controle. O ganho de peso foi similar entre os grupos intervenção e controle entre 0 e 6 meses (Média-M=4,57kg, Desvio-Padrão-DP $=0,90$ vs $M=4,56 \mathrm{~kg}, D P=1,03$ $p=0,918)$ e entre 6 e 12 meses $(M=1,96 \mathrm{~kg}$, $D P=0,81$ vs $M=4,56 \mathrm{~kg}, D P=1,97 p=0,821)$, e a ausência de efeito da intervenção sobre o desfecho do estudo permitiu que as crianças dos dois grupos fossem analisadas em conjunto.

Análises de frequência foram realizadas para descrição das variáveis categóricas e média e desvio-padrão para variáveis contínuas. As análises foram estratificadas por sexo. Comparou-se o ganho de peso médio entre 0 e 6 meses e entre 6 e 12 meses com o padrão estabelecido pelo estudo multicêntrico da OMS ${ }^{17}$ por meio do teste $t$ de Student para uma amostra. A avaliação do ganho de peso médio em relação às práticas alimentares foi realizada por meio do teste $t$ de Student para amostras independentes. Em todas as comparações, foi considerado um alfa crítico de 0,05 .

O projeto foi aprovado pelo Comitê de Ética de Pesquisa da Universidade Federal do Rio Grande do Sul sob o parecer de $n^{\circ} 200245$; todas as mães assinaram o Termo de Consentimento Livre e Esclarecido.

\section{RESULTADOS}

Dentre as 500 crianças inicialmente recrutadas para o estudo, 397 foram avaliadas aos 12 meses de idade. O principal motivo das perdas foi a não localização dos domicílios das crianças. Dentre essas crianças, 69 foram excluídas das análises aos 6 meses e 89 aos 12 meses, pois os questionários não apresentavam dados completos de peso e estatura no nascimento, aos 6 e aos 12 meses de idade. A amostra foi composta, portanto, de 308 crianças: 175 meninos e 133 meninas (perda 38,4\%, n=192). Algumas análises têm o número amostral menor do que o total devido à inconsistência ou à não coleta dos dados de algumas variáveis.

Em relação à renda familiar mensal: 6,2\% das famílias recebiam renda de até um salário- 
-mínimo; 47,0\%, renda entre um e três salários-mínimos; e 21,9\%, renda igual ou superior a três salários-mínimos. No momento da primeira entrevista, 79,3\% das mães viviam com seus companheiros, $80,3 \%$ tinham vinte anos ou mais e $58,2 \%$ tinham menos de oito anos de estudo. A prevalência de aleitamento materno exclusivo por menos de quatro meses foi de 65,2\%; desses, $16,9 \%$ receberam fórmulas infantis por, em média, 3,3 meses.

\section{Velocidade de ganho de peso do nascimento aos seis meses de idade}

A média de ganho de peso nos primeiros seis meses de vida foi de $M=4.663, D P=0,932 \mathrm{~kg}$ para os meninos e de $M=4.452, D P=1,007 \mathrm{~kg}$ para as meninas. Entre os meninos, a comparação da média de ganho de peso nos seis primeiros meses de vida com o padrão da OMS de $4.580 \mathrm{~kg}^{17}$ não mostrou diferença significativa $(p=0,225)$. As meninas, no entanto, apresentaram significativamente maior ganho de peso $(p=0,000)$ em relação ao padrão de ganho de peso da OMS de $4.079 \mathrm{~kg}^{17}$ (Tabela 1).

O consumo de leite de vaca adicionado de açúcar e farinha aos seis meses e a introdução precoce de açúcar - antes dos quatro meses de vida da criança - não foram associados com maior ganho de peso em ambos os sexos. A velocidade de ganho de peso nos primeiros seis meses de idade não foi estatisticamente diferente entre as crianças que receberam aleitamento materno exclusivo por até quatro meses ou mais e aquelas que foram amamentadas exclusivamente por período inferior a quatro meses (Tabela 1).

\section{Velocidade de ganho de peso dos seis aos doze meses de idade}

O ganho de peso médio entre 6 e 12 meses de idade foi de $M=1,929, D P=0,729 \mathrm{~kg}$ para os meninos e de $M=1,900, D P=0,881 \mathrm{~kg}$ para as meninas. O ganho de peso no segundo semestre de vida foi significativamente superior ao padrão da OMS $-1.688 \mathrm{~kg}$ para meninos e $1.618 \mathrm{~kg}$ para meninas $^{17}$-, para ambos os sexos $(p=0,001 \mathrm{e}$ $p=0,0001$ ) (Tabela 2).

O consumo precoce de açúcar de adição (antes dos quatro meses de idade) e o consumo de leite de vaca adicionado de açúcar e farinha aos 6 meses e aos 12 meses de idade não foram associados ao ganho de peso no segundo semestre de vida. O ganho de peso entre 6 e 12 meses de idade também não diferiu entre aqueles

Tabela 1. Ganho de peso no período de 0 a 6 meses, comparação com o padrão da Organização Mundial da Saúde e associação com práticas alimentares. São Leopoldo (RS), 2002-2003.

\begin{tabular}{|c|c|c|c|c|c|c|c|c|}
\hline \multirow{3}{*}{ Variáveis } & \multirow[b]{3}{*}{$\mathrm{n}$} & \multirow{2}{*}{\multicolumn{2}{|c|}{$\begin{array}{c}\text { Meninos } \\
\text { Ganho de peso }(\mathrm{kg})\end{array}$}} & \multirow[b]{3}{*}{$p$} & \multirow[b]{3}{*}{$\mathrm{n}$} & \multicolumn{2}{|c|}{ Meninas } & \multirow[b]{3}{*}{$p$} \\
\hline & & & & & & \multicolumn{2}{|c|}{ Ganho de peso $(\mathrm{kg})$} & \\
\hline & & Média & DP & & & Média & DP & \\
\hline Comparação com o padrão WHO* & 184 & 4.663 & 0,932 & 0,225 & 144 & 4.452 & 1,007 & 0,000 \\
\hline \multicolumn{9}{|c|}{ Consumo de leite de vaca com adição de açúcar e farinha aos 6 meses } \\
\hline Sim & 47 & 4.765 & 0,847 & 0,422 & 23 & 4.296 & 1,045 & 0,420 \\
\hline Não & 135 & 4.638 & 0,963 & & 121 & 4.481 & 1,001 & \\
\hline \multicolumn{9}{|l|}{ Consumo de açúcar de adição } \\
\hline$\leq 4$ meses & 98 & 4.749 & 0,992 & 0,120 & 80 & 4.522 & 0,991 & 0,232 \\
\hline$>4$ meses & 72 & 4.522 & 0,850 & & 50 & 4.311 & 0,945 & \\
\hline \multicolumn{9}{|l|}{ Aleitamento materno exclusivo } \\
\hline$<4$ meses & 108 & 4.722 & 0,969 & 0,351 & 82 & 4.462 & 1,020 & 0,718 \\
\hline$\geq 4$ meses & 67 & 4.585 & 0,888 & & 52 & 4.400 & 0,897 & \\
\hline
\end{tabular}

${ }^{*} 4.580 \mathrm{~kg}$ para os meninos e $4.079 \mathrm{~kg}$ para as meninas. DP: Desvio-Padrão. WHO: World Health Organization. 
560 | SC GONÇALVES et al.

Tabela 2. Ganho de peso no período de 6 a 12 meses, comparação com o padrão da Organização Mundial da Saúde e associação com práticas alimentares. São Leopoldo (RS), 2002-2003.

\begin{tabular}{|c|c|c|c|c|c|c|c|c|}
\hline \multirow{3}{*}{ Variáveis } & \multirow[b]{3}{*}{$\mathrm{n}$} & \multirow{2}{*}{\multicolumn{2}{|c|}{$\begin{array}{c}\text { Meninos } \\
\text { Ganho de peso }(\mathrm{kg})\end{array}$}} & \multirow[b]{3}{*}{$p$} & \multirow[b]{3}{*}{$\mathrm{n}$} & \multirow{2}{*}{\multicolumn{2}{|c|}{$\begin{array}{c}\text { Meninas } \\
\text { Ganho de peso }(\mathrm{kg})\end{array}$}} & \multirow[b]{3}{*}{$p$} \\
\hline & & & & & & & & \\
\hline & & Média & DP & & & Média & DP & \\
\hline Comparação com o padrão OMS* & 175 & 1.977 & 0,747 & 0,000 & 133 & 1.968 & 0,902 & 0,000 \\
\hline \multicolumn{9}{|c|}{ Consumo de leite de vaca com adição de açúcar e farinha aos 6 meses } \\
\hline Sim & 45 & 1.914 & 0,749 & \multirow[t]{2}{*}{0,495} & 22 & 2.243 & 0,829 & \multirow[t]{2}{*}{0,118} \\
\hline Não & 129 & 2.002 & 0,749 & & 111 & 1.913 & 0,910 & \\
\hline \multicolumn{9}{|c|}{ Consumo de leite de vaca com adição de açúcar e farinha aos 12 meses } \\
\hline Sim & 60 & 2.110 & 0,768 & \multirow[t]{2}{*}{0,096} & 35 & 2.014 & 0,891 & \multirow[t]{2}{*}{0,728} \\
\hline Não & 114 & 1.911 & 0,732 & & 98 & 1.951 & 0,910 & \\
\hline \multicolumn{9}{|c|}{ Consumo de refrigerantes aos 12 meses } \\
\hline Sim & 138 & 1.965 & 0,750 & \multirow[t]{2}{*}{0,696} & 101 & 1.944 & 0,877 & \multirow[t]{2}{*}{0,397} \\
\hline Não & 37 & 2.020 & 0,738 & & 26 & 2.112 & 0,990 & \\
\hline \multicolumn{9}{|l|}{ Consumo de açúcar de adição } \\
\hline$\leq 4$ meses & 98 & 1.989 & 0,786 & \multirow[t]{2}{*}{0,909} & 79 & 2.057 & 0,818 & \multirow[t]{2}{*}{0,120} \\
\hline$>4$ meses & 72 & 1.976 & 0,700 & & 50 & 1.801 & 1,030 & \\
\hline \multicolumn{9}{|l|}{ Aleitamento materno exclusivo } \\
\hline$<4$ meses & 108 & 2.077 & 0,777 & \multirow[t]{2}{*}{0,020} & 81 & 2.077 & 0,895 & \multirow[t]{2}{*}{0,080} \\
\hline$\geq 4$ meses & 67 & 1.814 & 0,669 & & 52 & 1.797 & 0,896 & \\
\hline
\end{tabular}

*1.688kg para os meninos e 1.618kg para as meninas. DP: Desvio-Padrão; OMS: Organização Mundial da Saúde.

que tinham consumido refrigerante no mês anterior à segunda entrevista em relação àqueles que não tinham consumido. As crianças do sexo masculino que receberam aleitamento materno exclusivo por período menor que quatro meses apresentaram ganho de peso superior ( $M=2.077$, $\mathrm{DP}=0,777 \mathrm{~kg}$ ) às crianças que receberam somente leite materno por período igual ou superior a quatro meses $(\mathrm{M}=1.814, \mathrm{DP}=0,669 \mathrm{~kg})(p=0,020)$. Entre as meninas, o grupo que recebeu leite materno exclusivamente por tempo inferior a quatro meses apresentou ganho de peso superior $(\mathrm{M}=2.077, \mathrm{DP}=0,895 \mathrm{~kg})$ quando comparado ao grupo que foi amamentado exclusivamente por mais tempo $(M=1.797, D P=0,896 \mathrm{~kg})$, porém a estatística não alcançou valor crítico que permita afastar a hipótese nula $(p=0,080)$ (Tabela 2).

\section{I S C U S S Ã O}

Os resultados deste estudo mostraram que as crianças avaliadas apresentaram ganho de peso excessivo no primeiro ano de vida em relação ao padrão da OMS e que, entre os meninos, o aleitamento materno exclusivo por período igual ou superior a quatro meses foi associado a menor ganho de peso entre 6 e 12 meses de idade.

Um dos fatores que pode ter contribuído para a alta velocidade de ganho de peso dessa amostra é a condição socioeconômica desfavorável dessa população, a qual pode ser observada na análise dos dados de renda familiar e escolaridade materna. Grande parte das famílias avaliadas vive em moradias caracterizadas por condições ambientais e sanitárias precárias, e estudos indicam que moradores de favelas urbanas tendem a ter indicadores de saúde muito aquém do recomendado ${ }^{21,22}$. Estudos internacionais apontam a existência de maior risco de ganho de peso excessivo entre as pessoas de baixo nível socioeconômico em comparação àquelas com melhores condições econômicas ${ }^{23,24}$. Estudos populacionais nacionais não mostram essa tendência de forma tão estrita ${ }^{25}$, mas evidências recentes sugerem que, independentemente das diferenças 
nas taxas de excesso de peso, as suas consequências para a saúde são mais severas entre os mais pobres ${ }^{26}$.

Evidências concretas enfatizam as consequências negativas do ganho de peso excessivo no primeiro ano de vida e sua importância no desenvolvimento de sobrepeso e obesidade posteriores ${ }^{8-10,27}$. Ademais, Holzhauer et al. ${ }^{11}$ relataram que crianças que ganharam peso de forma rápida no primeiro ano de vida apresentaram desenvolvimento desigual dos tecidos do corpo, pois adquiriram quantidades relativamente maiores de gordura distribuída na região abdominal, o que pode estar associado a doenças crônicas na vida adulta.

A prática de aleitamento materno exclusivo por período igual ou superior a quatro meses mostrou-se significativamente associada ao menor ganho de peso entre 6 e 12 meses de idade entre os meninos. Estudo de Rzehak et al. ${ }^{28}$ também observou efeito protetor do aleitamento sobre a velocidade de ganho de peso nos doze primeiros meses de vida, o qual prosseguiu até os seis anos de idade. Esse resultado pode ter implicações práticas ainda mais relevantes ao se considerar que os padrões de peso na infância tendem a se manter até a vida adulta. Moran ${ }^{29}$ sugeriu que, a partir dos três anos de idade, o excesso de peso torna-se definitivamente determinante de obesidade futura, e se a criança é obesa aos seis anos de idade ela apresenta 50,0\% de chances de tornar-se um adulto obeso ${ }^{29}$. Estudo recente encontrou resultados semelhantes, mostrando que $40,0 \%$ dos meninos e $48,6 \%$ das meninas que apresentavam obesidade aos 18 anos já eram obesos aos sete anos de idade ${ }^{30}$.

Uma hipótese para justificar o impacto positivo do leite materno sobre o ganho de peso é a sua influência sobre a concentração hormonal dos recém-nascidos ${ }^{14,15,28}$. Savino et al. ${ }^{31}$ demonstrou que crianças que recebiam leites artificiais tinham níveis mais elevados de grelina e Fator de Crescimento Insulina Tipo 1 (IGF-1) e menores níveis de leptina aos quatro meses de vida, o que sugere que o leite humano poderia mediar o controle da saciedade nas crianças que o conso- mem, diminuindo o apetite em comparação às desmamadas. Outros autores ${ }^{32,33}$ também relataram maiores ganhos de peso com alimentação artificial em comparação ao leite materno, mas alertaram para a hipótese de que a proteção do leite materno para o ganho de peso excessivo pode não ocorrer por seus próprios efeitos, mas pela não utilização de outros leites não humanos, que, com maiores teores de proteína, ativariam maior resposta do hormônio IGF-1 e secreção de insulina e diminuiriam a secreção do hormônio do crescimento, levando a um consequente maior aumento do peso. Essa justificativa aplica-se à população estudada, pois se sabe que grande proporção de crianças que param de receber leite materno passa a consumir leite de vaca e outros alimentos complementares ${ }^{34}$ com elevada quantidade de proteína quando comparados ao leite materno.

Salienta-se que os resultados apresentados não podem ser extrapolados para crianças nascidas com baixo peso (<2.500g), as quais não fizeram parte da amostra do estudo, já que elas podem apresentar outros padrões de ganho de peso e responder de forma distinta aos fatores nutricionais e ambientais às quais são expostas.

\section{O N C L U S Ã O}

Os resultados deste estudo evidenciaram que as crianças avaliadas apresentaram ganho de peso excessivo no primeiro ano de vida e que o aleitamento materno exclusivo apresentou papel protetor para os meninos. Salienta-se a importância de ações efetivas para promover a prática de aleitamento materno e a prevenção no ganho de peso acelerado na atenção primária à saúde.

\section{COLABORA D ORES}

SC GONÇALVES e ML LOUZADA participaram da concepção do artigo, análise e interpretação dos dados, revisão crítica do artigo e aprovação final da versão a ser publicada. PD CAMPAGNOLO participou na coleta de dados, concepção, delineamento e revi- 
são crítica do artigo e da aprovação final da versão a ser publicada. MR VITOLO participou na coordenação e delineamento do estudo maior, concepção e delineamento do artigo, revisão crítica e aprovação final da versão a ser publicada.

\section{REFERÊ NCIAS}

1. World Health Organization. Diet, nutrition and the prevention of chronic diseases. Geneva: WHO; 2003.

2. de Onis M, Blossner M, Borghi E. Global prevalence and trends of overweight and obesity among preschool children. Am J Clin Nutr. 2010; 92(5): 1257-64. doi: 10.3945/ajen.2010.29786.

3. Brasil. Ministério da Saúde. Pesquisa nacional de demografia e saúde da criança e da mulher. Brasília: Ministério da Saúde; 2008.

4. Vitolo MR, Gama CM, Bortolini GA, Campagnolo PD, Drachler ML. Some risk factors associated with overweight, stunting and wasting among children under 5 years old. J Pediatr. 2008; 84(3):251-57. doi:10.2223/JPED.1776.

5. Monteiro CA, Conde WL. Tendência secular da desnutrição e da obesidade na infância na cidade de São Paulo (1974-1996). Rev Saúde Pública. 2000; 34(Suppl 6):52-61. doi: 10.1590/S0034-891020 00000700008

6. Gigante DP, Victora CG, Araújo CL, Barros FC. Tendências no perfil nutricional das crianças nascidas em 1993 em Pelotas, Rio Grande do Sul, Brasil: análises longitudinais. Cad Saúde Pública. 2003; 19 (Suppl 1):141-7. doi: 10.1590/S0102-311X20 03000700015 .

7. Menezes RC, Lira PI, Oliveira JS, Leal VS, Santana SC, Andrade SL, et al. Prevalence and determinants of overweight in preschool children. J Pediatr. 2011; 87(3):231-7. doi:10.2223/JPED.2092.

8. Dennison BA, Edmunds LS, Stratton HH, Pruzek RM. Rapid infant weight gain predicts childhood overweight. Obesity (Silver Spring). 2006; 14(3): 491-9. doi: 10.1038/oby.2006.64.

9. Matos SMA, Jesus SR, Saldiva SRDM, Prado MS, D'Innocenzo S, Assis AMO, et al. Velocidade de ganho de peso nos primeiros anos de vida e excesso de peso entre 5-11 anos de idade, Salvador, Bahia, Brasil. Cad Saúde Pública. 2011; 27(4):714-22. doi: 10.1590/S0102-311X2011000400010.

10. Botton J, Heude B, Maccario J, Ducimetiere P, Charles MA. Postnatal weight and height growth velocities at different ages between birth and $5 \mathrm{y}$ and body composition in adolescent boys and girls. Am J Clin Nutr. 2008; 87(6):1760-8.

11. Holzhauer S, Hokken Koelega AC, Ridder $M$, Hofman A, Moll HA, Steegers EA, et al. Effect of birth weight and postnatal weight gain on body composition in early infancy: the generation $\mathrm{R}$ study. Early Hum Dev. 2009; 85(5):285-90. doi:10.1016/j.earlhumdev.2008.11.002.

12. Larnkjaer A, Schack-Nielsen L, Molgaard C, Ingstrup HK, Holst JJ, Michaelsen KF. Effect of growth in infancy on body composition, insulin resistance, and concentration of appetite hormones in adolescence. Am J Clin Nutr. 2010; 91(6):1675-83. doi: 10.3945/ajcn.2009.27956.

13. Hendrie GA, Coveney J, Cox DN. Defining the complexity of childhood obesity and related behaviours within the family environment using structural equation modelling. Public Health Nutr. 2012: 15(1):48-57. doi: 10.1017/S1368980011 001832.

14. Apfelbacher C, Loerbroks A, Cairns J, Behrendt H, Ring J, Krämer U. Predictors of overweight and obesity in five to seven-year-old children in Germany: results from cross-sectional studies. BMC Public Health. 2008; 8:171. doi: 10.1186/1471-2 458-8-171.

15. Silva MV, Ometto AMH, Furtuoso COM, Pipitone MAP, Sturion GL. Acesso à creche e estado nutricional das crianças brasileiras: diferenças regionais, por faixa etária e classes de renda. Rev Nutr. 2000; 13(3):193-9. doi: 10.1590/S1415-52732000000 300006.

16. Koletzko B, von Kries R, Closa R, Escribano J, Scaglioni S, Giovannini M, et al. Can infant feeding choices modulate later obesity risk? Am J Clin Nutr. 2009; 89(5):1502-8. doi: 10.3945/ajcn.2009.27 $113 \mathrm{D}$.

17. World Health Organization. The WHO Multicentre Growth Reference Study (MGRS): child growth standard. Geneva: WHO; 2006.

18. de Onis M, Garza C, Victora CG, Onyango AW, Frongillo EA, Martines J. The WHO Multicentre Growth Reference Study: planning, study design, and methodology. Food Nutr Bull. 2004; 25(Suppl 1):15-26.

19. Vitolo MR, Bortolini GA, Feldens CA, Drachler ML. Impactos da implementação dos dez passos da alimentação saudável para crianças: ensaio de campo randomizado. Cad Saúde Publica. 2005; 21(5):1448-57. doi: 10.1590/S0102-311X20050 00500018

20. World Health Organization. Global Strategy for infant and young child feeding. Geneva: WHO; 2006. 
21. Szwarcwald $C L$, Bastos Fl, Esteves MA, Andrade $C L$, Paez MS, Medici EV, et al. Desigualdade de renda e situação de saúde: o caso do Rio de Janeiro. Cad Saúde Pública. 1999; 15(1):15-28. doi: 10.15 90/S0102-311X1999000100002.

22. de Snyder VN, Friel S, Fotso JC, Khadr Z, Meresman $S$, Monge $P$, et al. Social conditions and urban health inequities: realities, challenges and opportunities to transform the urban landscape through research and action. J Urban Health. 2011. 88(6):1183-93. doi: 10.1007/s11524-011-9609-y.

23. Wijlaars $L P$, Johnson $L$, van Jaarsveld $C H$, Wardle J. Socioeconomic status and weight gain in early infancy. Int J Obes. 2011; 35(7):963-70. doi:10.1 038/ijo.2011.88.

24. Murasko JE. Trends in the associations between family income, height and body mass index in US children and adolescents: 1971-1980 and 1999-2008. Ann Hum Biol. 2011; 38(3):290-306. doi: 10.310 9/03014460.2010.537698.

25. Instituto Brasileiro de Geografia e Estatística. Pesquisa de orçamentos familiares 2008-2009. Rio de Janeiro: IBGE; 2010.

26. Jolliffe D. Overweight and poor? On the relationship between income and the body mass index. Econ Hum Biol. 2011; 9(4):342-355. doi:10.1016/j.ehb. 2011.07.004.

27. Monasta L, Batty GD, Cattaneo A, Lutje V, Ronfani L, Van Lenthe FJ, et al. Early-life determinants of overweight and obesity: a review of systematic reviews. Obes Rev. 2010; 11(10):695-708. doi: 10.1 111/j.1467-789X.2010.00735.x.

28. Rzehak $P$, Sausenthaler $S$, Koletzko S, Bauer $C P$, Schaaf B, von Berg A, et al. Period-specific growth, overweight and modification by breastfeeding in the GINI and LISA birth cohorts up to age 6 years. Eur J Epidemiol. 2009; 24(8):449-67. doi: 10.1111/j. 1467-789X.2010.00735.x.

29. Moran R. Evaluation and treatment of childhood obesity. Am Fam Physician. 1999; 59(4):8.

30. Starc G, Strel J. Tracking excess weight and obesity from childhood to young adulthood: a 12-year prospective cohort study in Slovenia. Public Health Nutr. 2011; 14(1):49-55. doi: 10.1017/\$1368980 010000741.

31. Savino F, Fissore MF, Grassino EC, Nanni GE, Oggero R, Silvestro L. Ghrelin, leptin and IGF-I levels in breast-fed and formula-fed infants in the first years of life. Acta Paediatr. 2005; 94(5):531-7.

32. Ong KK, Langkamp M, Ranke MB, Whitehead K, Hughes IA, Acerini CL, et al. Insulin-like growth factor I concentrations in infancy predict differential gains in body length and adiposity: the cambridge baby growth study. Am J Clin Nutr. 2009; 90(1): 156-61. doi: 10.3945/ajcn.2008.27408.

33. Schack-Nielsen L, Sorensen T, Mortensen EL, Michaelsen KF. Late introduction of complementary feeding, rather than duration of breastfeeding, may protect against adult overweight. Am J Clin Nutr. 2010; 91(3):619-27. doi: 10.3945/ajen.2008.27 078.

34. Caetano MC, Ortiz TT, Silva SG, Souza FI, Sarni RO. Complementary feeding: inappropriate practices in infants. J Pediatr. 2010; 86(3):196-201. doi: 10.1590/S0021-75572010000300006.

Recebido em: 26/9/2011 Versão final em: 20/6/2012 Aprovado em: 11/7/2012 
